\title{
Nonsurgical Treatment of Class III Malocclusion with Nonextraction and Extraction of Mandibular First Premolar
}

\author{
Nazruddin \\ Department of Orthodontics \\ Faculty of Dentistry, Universitas Sumatera Utara \\ Medan, Indonesia
}

\begin{abstract}
Skeletal Class III malocclusion is some of the most complex cases to treat. Currently, orthognathic surgery and orthodontic camouflage are the standard technique for treating this condition in fully growing patients. With skeletal Class III orthodontic patients camouflage can be used to treat milder cases with Class III elastic. When patient declines orthodontic surgery, camouflage treatment with Class III elastics is a valid option. In this case report will showing two patients with skeletal Class III and the treatment for the first patient using fixed appliance and Class III elastic, the second patient using camouflage treatment and extraction of both the mandibular first premolars, and Class III elastic. The result of treatment are for the first patient malocclusion could be treated with Class III elastic and orthodontic movement, and the second patient the treatment technique needed extraction of the mandibular first premolar and used Class III elastic. As a conclusion are the skeletal Class III malocclusion could be treated with or without extraction of mandibular first premolar and the treatment have to use Class III elastics.
\end{abstract}

Keywords-skeletal class III malocclusion, class III elastic, camouflage treatment

\section{INTRODUCTION}

The prevalence of skeletal class III malocclusion varies among races. In the white population, the incidence has been reported to be $1 \%$ to $5 \%$, and in the Asian populations it is up to $14 \%$. This malocclusion is considered one of the most difficult to treat. Class III treatment is considerable clinical challenge and commonly includes (1) growth modification involving a chin cup to restrain mandibular growth or a facemask to protract the maxilla, (2) dentoalveolar compensation or camouflage involving dental extractions, and (3) orthognathic surgery. In this list alternatives orthodontic treatment is often seen as either a less-desirable alternative to surgery or a treatment reserved for milder skeletal problems [1,2,3].

Nongrowing patients with skeletal Class III malocclusion could be treated by orthognathic surgery or orthodontic camouflage treatment. In severe cases, surgical procedures should be performed to correct the skeletal and dental discrepancies, as well as to improve facial esthetics and harmonize the profile $[2,4,5]$.
In borderline cases where camouflage is possible and is a valid option, the treatment should camouflage the dental and skeletal discrepancies to an extent that could satisfy functional concerns of the patient as much as possible. This is sometimes difficult to achieve due to lack of adequate anchorage since adequate and proper anchorage control is fundamental to the success of orthodontic treatment [5,6,7].

Skeletal Class III malocclusion, are some of the most complex cases to treat. Currently Orthognathic surgery and orthodontic camouflage are the standard techniques for treating this condition in fully growing patients. Although the surgical approach is aimed at improving their facial esthetics and correcting their skeletal and dental discrepancies, the surgical correction might not result in enough change in the facial profile of borderline patients. Camouflage treatment may not give the desired outcome $[4,5,8]$.

With skeletal Class III patient, orthodontic camouflage can be used to treat milder cases with Class III elastics, but it is limited to patients with a low mandibular plane angle and a deep bite. This method is therefore unacceptable for most Class III patients who have high mandibular plane angles and increased lower anterior facial heights. Class III elastics extrude the maxillary molars and cause clockwise mandibular rotation that result in increased lower anterior facial height. Furthermore, Class III elastics exacerbate the already proclined maxillary anterior teeth, compromising both esthetics and overall stability of treatment. Even so, when the patient declines orthognathic surgery, camouflage treatment with Class III elastics is a valid option [4,6,7,8,9].

Camouflage orthodontic treatment can be considered to correct mild skeletal Class III patients with decent profiles. However, when the skeletal Class III discrepancy is beyond the limit of dental compensation, orthognathic surgery may be the only option for creating a stable occlusion. The exact point where only orthodontic treatment was needed is still debatable $[7,8,9]$. 


\section{CASE REPORT}

This case report represents two cases of Class III malocclusion with different ages and different anomaly condition.

\section{A. Case 1}

The patient is 13 years-old female wants to treat her teeth because there are crowded teeth in the maxilla and could not bite with normal in the anterior side. Extraoral examination the patient has concave profile. Intra-oral examination there are anterior crossbite, crowding anterior teeth, shifting of median line, ectopic position of both maxillary canines, and molar and canine relationship are Class III Angle. Oral hygiene is good.

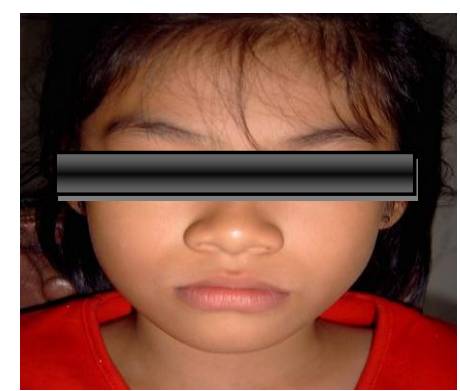

Figure 1. Photo profile of patient from anterior.

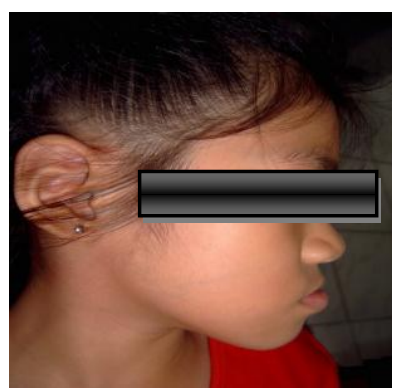

Figure 2. Photo profile from lateral.

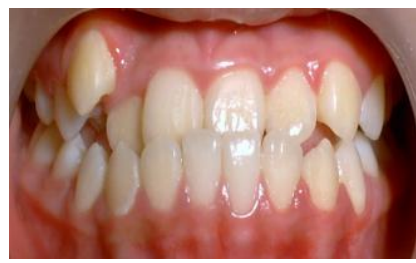

Figure 3. Intra-oral photo of patient from frontal. There are severe crowding in maxillary teeth, ectopic position of right canine anterior crossbite and shifting of midline.

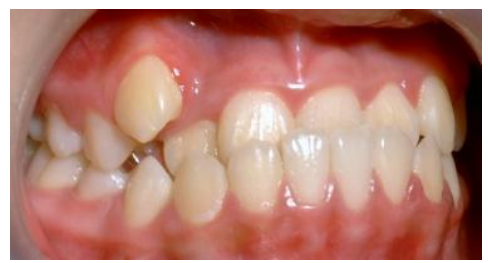

Figure 4. Intra-oral photo from right side. There are anterior crowded in maxilla, ectopic right upper canine, Class III molar relationship, and extrusive of mandibular anterior teeth.

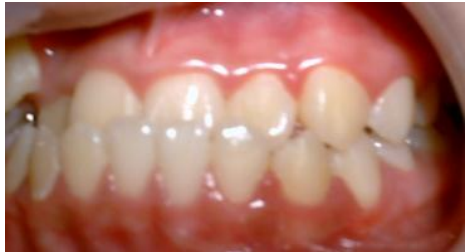

Figure 5. Intra oral photo from left side, showing anterior crossbite, ectopic left canine and Class III molar relationship.

\section{B. Case 2}

The patient is a 34 years-old female sought treatment for correction of abnormal teeth relationship. Extra oral examination showed concave profile. Facial aesthetic evaluation revealed slight mandibular prognation and lower lip protrusion. Intra oral examination, patient presented deficiency of maxilla in which maxillary arch was smaller than mandibular arch, midline slight shifting. Crossbite relationship extended from anterior to posterior. Molar and canine relation is Class III Angle. Patient has good oral hygiene.

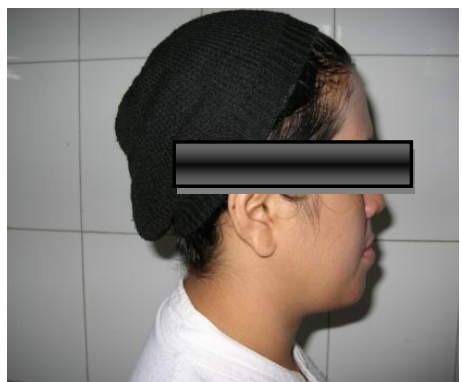

Figure 6. Photo profile of case 2 patient from lateral.

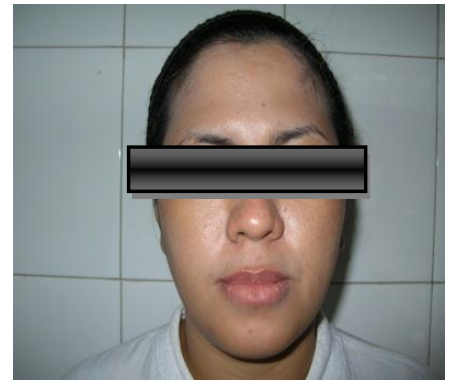

Figure 7. Photo profile of case 2 from anterior.

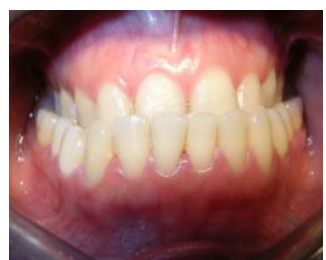

Figure 8. Intra-oral photo of case 2 from anterior representing Clas III skeletal with crossbite from anterior to posterior before treatment.

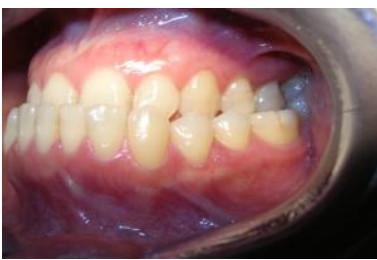

Figure 9. Intra-oral photo of case 2 from left side before treatment. 


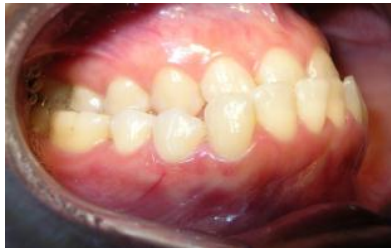

Figure 10. Intra-oral photo of case 2 from right side before treatment.

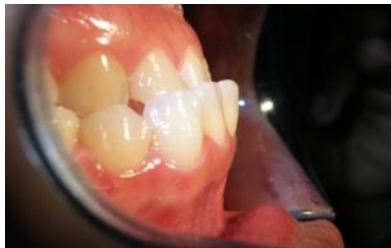

Figure 11. Intra-oral photo of case 2 representing position of overjet and overbite before treatment.

\section{CASE MANAGEMENT}

\section{A. Treatment objectives}

The treatment objectives are depends to the type of malocclusion of each patient.

The patient of case 1 is in growing case, included non-extraction treatment, correction of crowded, crossbite, overjet and overbite, midline deviation, achieving Class I molar and canine relationship, eliminating the anterior functional shift and establishing canine guidance, improving facial appearance.

The patient of case 2 the patient is non-growing case, included camouflage treatment, extraction of both mandibular first premolars, correction of crossbite, overjet and overbite, midline deviation, to expand maxillary ach and to decreased the width of mandibular arch, and molar relationship still Class III.

\section{B. Treatment progress}

- Case 1

The malocclusion was treated with .022 in edgewise bracket. After bracket setting the treatment was started with initial leveling and aligning with inserted .014 Regency archwire in both arch. And in the next visit the wire was changed to .016 Regency archwire until anterior crowding corrected. In this time the patient was begun wear light Class III elastic $(2.5 \mathrm{oz})$. And then archwire in the both arch was changed to .018 SS and with molar offset and tipback bend 40 degree in the molars region. Class III elastic still continue to use and the elastic force was changed to medium $(4.5 \mathrm{oz})$, until crossbite and midline corrected. In the next visit archwire was changed to .016x.025 SS as ideal arch and to use another type of elastic to find good interdigitation. The total treatment time is 27 months. Removable retainers were provided for night-time wear.

The results of treatment, crossbite, crowding, and midline were corrected; molar and canine became Class I relationship, and had good profile. The patient and her parent were satisfied with the results of treatment.

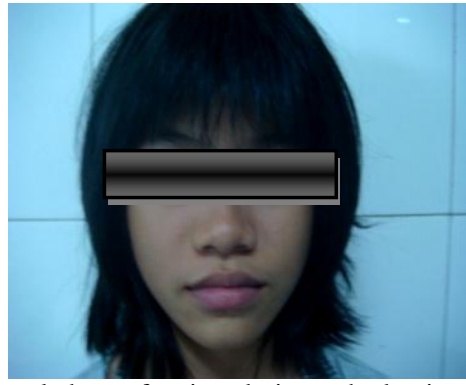

Figure 12. Frontal photo of patient during orthodontic treatment

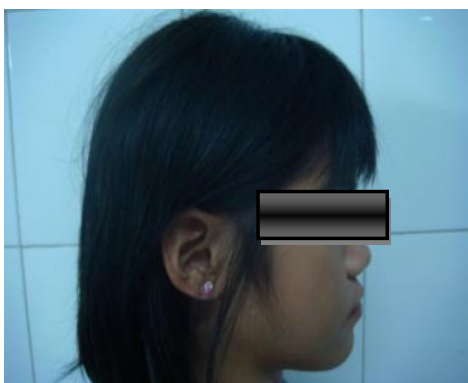

Figure 13. Lateral photo of patient during orthodontic treatment.

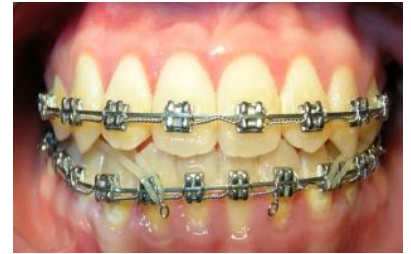

Figure 14. Intra-oral photo from frontal showing that maxillary anterior crowded, ectopic canines, midline and anterior crossbite were corrected.

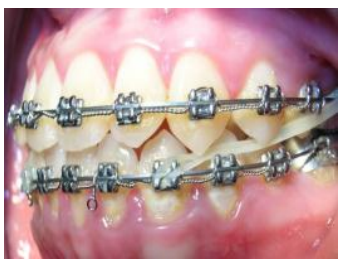

Figure 15. Intra oral photo from left side showing patient using Class III elastic and molar and canine relationship were corrected to Class I.

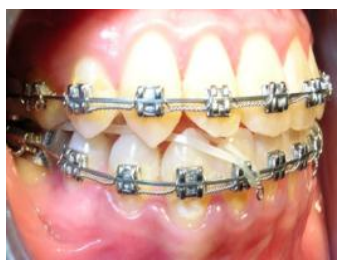

Figure 16. Intra oral photo from right side showing molar and canine were corrected to Class I relationship.

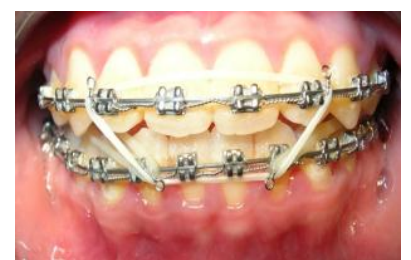

Figure 17. Intra-oral photo from frontal showing patient wearing two type of elastic, one Class III elastic and another one box type elastic to find good interdigitation in anterior side. 


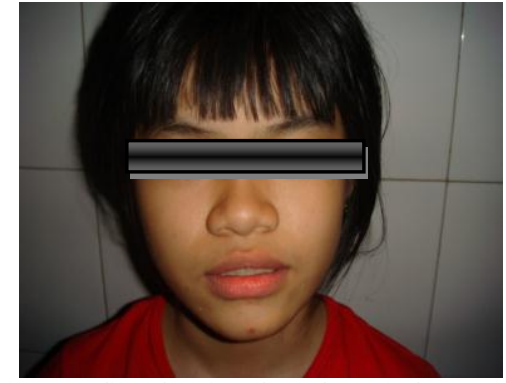

Figure 18. Photo profile of patient from frontal after treatment.

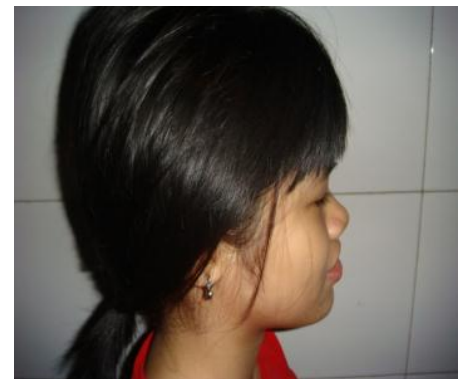

Figure 19. Photo profile of patient from lateral after treatment.

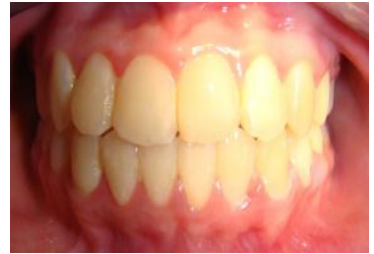

Figure 20. Intra-oral photo of patient from frontal after treatment, all of anomaly were corrected

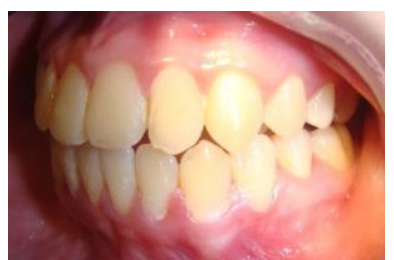

Figure 21. Intra-oral photo of patient from left side after treatment.

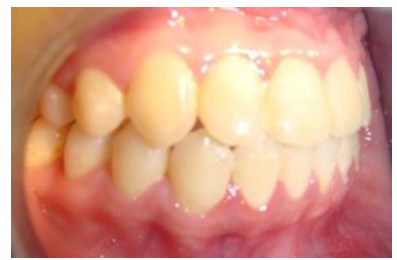

Figure 22. Intra-oral photo of patient from right side after treatment.

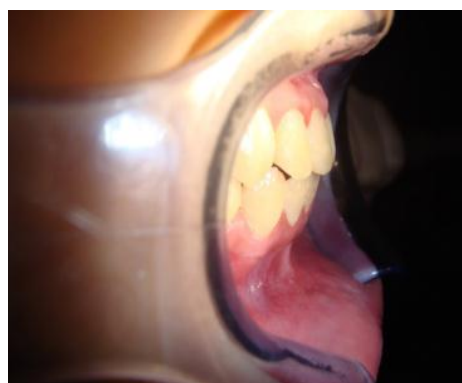

Figure 23. Intra-oral photo representing overjet and overbite after treatment.

\section{- $\quad$ Case 2}

The malocclusion was treated with .022 edgewise bracket. Type of treatment was to use camouflage treatment technique with extraction of both mandibular first premolar and using Class III elastic.

The treatment was started with extraction of both mandibular first premolars in preparation for comprehensive treatment. After bracket setting, the .014 in Regency archwire was inserted to bracket for initial leveling and aligning. In the next visit the wire was changed to .016 in Regency archwire. After leveling and aligning the wire was changed with more hard type wire to .018 SS and with offset and tipback 40 degree in molars region. Class III elastic was used in this time. In the next visit in Maxilla the archwire was changes to $0.017 \times 0.025$ Regency archwire with broad arch archwire type to expand maxilla and in mandibular the retraction of canine was began until Class I canine relationship achieved. And the wire was changed with 0.016x.025 SS archwire with standard type of archwire. In the next treatment was to retract mandibular anterior teeth, and Class III elastic still using until mandibular anterior crossbite corrected. The results of treatment, crossbite and midline corrected, and canine had Class I relationship and the patient had good profile, the molar still Class III relationship. Removable retainers were provided for night-time wear. In the next photograph below will showing the photos of first patient during treatment and after treatment.

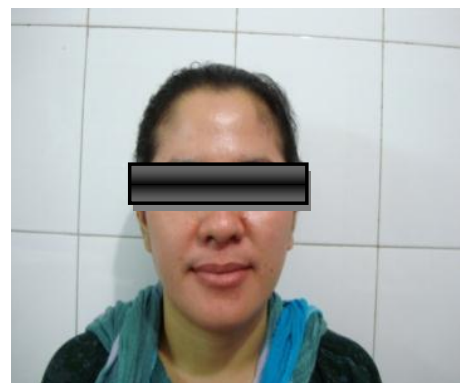

Figure 24. Photo profile from lateral during treatment.

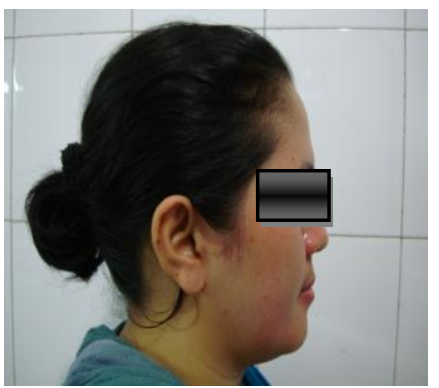

Figure 25. Photo profile from lateral during treatment.

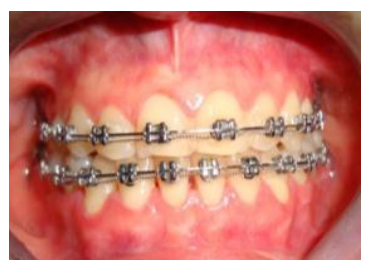

Figure 26. Intra-oral photo from anterior during treatment 


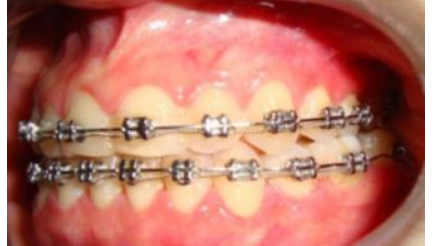

Figure 27. Intra-oral photo from left side during treatment.

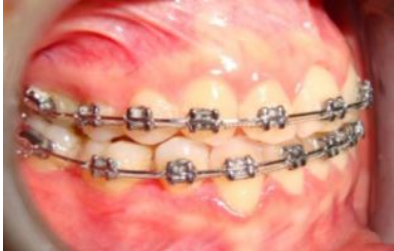

Figure 28. Intra-oral photo from right side during treatment.

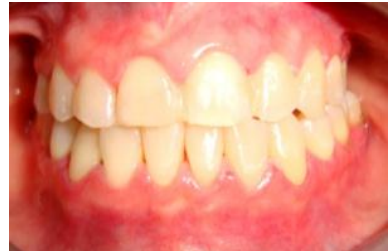

Figure 29. Intra-oral frontal photograph represented teeth posttreatment.

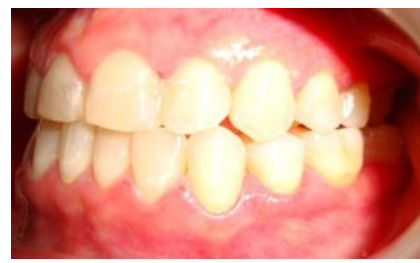

Figure 30. Intra-oral lateral photograph from left side after treatment.

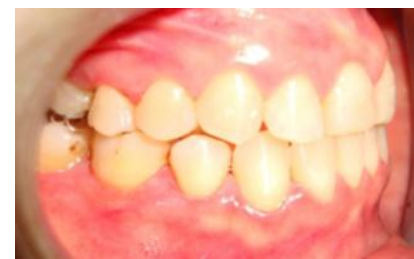

Figure 31. Intra-oral lateral photograph from right side after treatment.

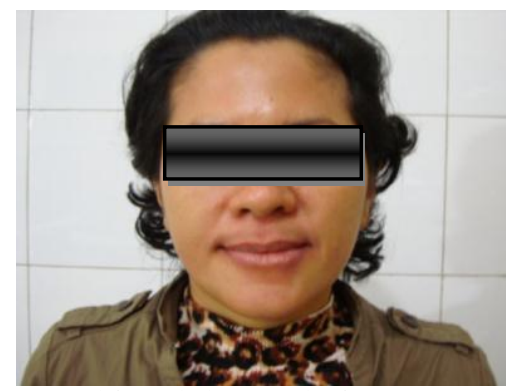

Figure 32. Extra-oral frontal photograph after treatment.

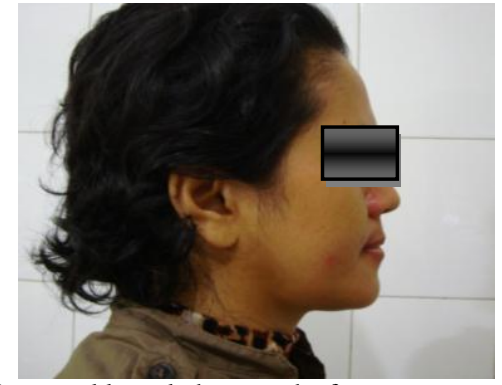

Figure 33. Extra-oral lateral photograph after treatment.

\section{DISCUSSION}

For most practitioners, the small percentage of Class III treatment represents large clinical challenges. Protraction facemask therapy has been advocated in the early treatment of Class III malocclusions with maxillary deficiency. However efforts to restraint mandibular growth at an early age rarely succeed because later mandibular growth often negated early correction. Most facemask patients significantly improved in the short term, but current data suggest that proximally $25 \%$ eventually require orthognathic surgery anyway. Better selection of patients for facemask treatment should improve the effectiveness and efficiency of the method $[2,3,4,10]$.

Orthodontic surgical correction is typically recommended to non-growing patients with larger dentoskeletal Class III discrepancies not amenable to orthodontic camouflage. Dentoalveolar compensation, or camouflage treatment can be viable alternative for non-growing patients with milder Class III discrepancies. It should be emphasized that one should not commit to camouflage treatment in growing patients, with progressive Class III deformities $[5,8,9,11]$.

In the first case represented that in the growing patient (case 1), Class III maxillary malocclusion could be treated with fixed appliance and used Class III elastic. To use Class III elastic is needed good cooperation from patient and her parent, because without have good cooperation the good results of treatment with to use Class III elastic will not achieved.

In the second case, patient is non-growing stage; therefore in this case treatment had been used camouflage treatment technique and extraction of both mandibular first premolars and wearing Class III elastic. She had good results of treatment in which all of anomaly condition were corrected, exception molar relationship still Class III.

Class III malocclusion could be treated in the growing and non-growing patients. Good cooperative in wearing Class III elastics are very important in the treatment of Class III malocclusion, because without have good cooperation of the patients and parent, the good results of treatment will be not achieved.

\section{REFERENCES}

[1] N.R. Burns NR, "Class III camouflage treatment: What are the limits?" Am. J. Orthod. Dentofacial Orthop., vol. 137(9), pp. 1$13,2010$. 
[2] R.M. Diego, "Camouflage of moderate class III malocclusions with extraction of lower second molar and mandibular cervical headgear," vol. 8(2), pp. 300-307, 2007.

[3] H. Hu, J. Chen, et al., "Distalization of the mandibular dentition of an adult with skeletal class III malocclusion," Am. J. Orthod. Dentofacial Orthop., vol. 142, pp. 854-862, 2012.

[4] Y. Jing, et al., "Nonsurgical correction of a class III malocclusion in adult by miniscrew assisted mandibular dentition distalization.” Am. J. Orthod. Dentofacial Orthop., vol. 143, pp. 877-887, 2013.

[5] L. Khan, et al., "Orthodontic camouflage treatment of class III malocclusion in non-growing patient," Orthod. J. of Nepal, vol. 5(1), pp. 45-48.

[6] A.T. Moullas, "Nonsurgical treatment of a patient with a class III malocclusion," Am. J. Orthod. Dentofacial Orthop., vol. 129, pp. 111-118, 2006
[7] S. Sato, "Case report: developmental characterization of skeletal class III malocclusion,” Angle Orthod., vol. 64(2), pp. 105 -111, 1994.

[8] E. Stellzig, "Treatment decision in adult patients with class III malocclusion: orthodontic therapy or orthoganthic surgery," Am. J. Orthod. Dentofacial Orthop., vol. 122, pp. 306-320, 2002.

[9] S. Timothy, "Orthodontic camouflage," Am. J. Orthod. Society, 31-34, 2006.

[10] Y.J. Soo, et al., "Camouflage treatment of skeletal class III malocclusion with asymmetry using a bone-borne rapid maxillary expander," Angle Orthop., vol. 85(2), pp. 323-333, 2015.

[11] P.Y. Weswood, "Long-term effects of class III treatment with RME and facemask therapy followed by fixed appliances," Am J. Orthod. Dentofacial Orthop., vol. 123, pp. 306-320, 2003. 